\title{
An Implicit Plan Overrides an Explicit Strategy during Visuomotor Adaptation
}

\author{
Pietro Mazzoni and John W. Krakauer \\ Motor Performance Laboratory, Department of Neurology, Columbia University College of Physicians and Surgeons, New York, New York 10032
}

The relationship between implicit and explicit processes during motor learning, and for visuomotor adaptation in particular, is poorly understood. We set up a conflict between implicit and explicit processes by instructing subjects to counter a visuomotor rotation using a cognitive strategy in a pointing task. Specifically, they were told the exact nature of the directional perturbation, a rotation that directed them $45^{\circ}$ counterclockwise from the desired target, and they were instructed to counter it by aiming for the neighboring clockwise target, $45^{\circ}$ away. Subjects were initially successful in completely negating the rotation with this strategy. Surprisingly, however, they were unable to sustain explicit control and made increasingly large errors to the desired target. The cognitive strategy failed because subjects simultaneously adapted unconsciously to the rotation to the neighboring target. Notably, the rate of implicit adaptation to the neighboring target was not significantly different from rotation adaptation in the absence of an opposing explicit strategy. These results indicate that explicit strategies cannot substitute for implicit adaptation to a visuomotor rotation and are in fact overridden by the motor planning system. This suggests that the motor system requires that planned and executed trajectories remain congruous in visual space, and enforces this correspondence even at the expense of an opposing explicit task goal.

Key words: adaptation; motor intention; motor learning; wrist; control; visuomotor rotation

\section{Introduction}

There has been much recent interest in the role of explicit processes during sequence learning (Willingham, 2001), where there is evidence that they can contribute to learning (Nissen and Bullemer, 1987; Reber and Squire, 1998; Sakai et al., 2003). In contrast, the role of explicit processes in motor adaptation has been less studied. Indirect evidence against a role for explicit processes in motor adaptation comes from studies that indicate that mirror drawing (Gabrieli et al., 1993), rotor pursuit (Tranel et al., 1994), and force field learning (Shadmehr et al., 1998) are accomplished at the same rate with and without declarative memory. In addition, it has been shown that visuomotor rotation learning can proceed without subject awareness (Kagerer et al., 1997; Hatada et al., 2005; Klassen et al., 2005).

The question whether motor learning can be purely implicit is different from the question whether explicit processes can contribute to motor learning. The first question has been addressed with dual-task designs, in which implicit learning is defined as a process that is nonintentional and automatic (Frensch, 1998) and thus should not be interfered with by the need to attend to a second task (Redding et al., 1992; Frensch, 1998). The second

Received Dec. 13, 2005; revised Feb. 18, 2006; accepted Feb. 21, 2006.

This work was supported by National Institute of Neurological Disorders and Stroke Grant K02 NS048099. We thank Sophie Ryan and Johnny Liang for technical assistance and Robert Sainburg for sharing experimental software. The wrist-pointing task was designed in collaboration with Claude Ghez.

Correspondence should be addressed to Dr. John W. Krakauer, Motor Performance Laboratory, Department of Neurology, Columbia University College of Physicians and Surgeons, 710 West 168th Street, New York, NY 10032. E-mail: jwk18@columbia.edu.

DOI:10.1523/JNEUROSCI.5317-05.2006

Copyright $\odot 2006$ Society for Neuroscience $\quad$ 0270-6474/06/263642-04\$15.00/0 question, which is the topic of this report, has been addressed by comparing the rate or degree of learning in subjects who are aware of the task structure versus those who are not aware of it (Willingham et al., 1989). There are a number of limitations to this approach, however. First, in the absence of specific instructions, subjects may vary in how they use explicit information. Second, motor performance might change with awareness rather than implicit learning per se. Third, different mixtures of implicit and explicit processes could lead to the same rate of learning. Therefore, we adopted a different approach in which we assessed the role of explicit and implicit processes by placing them in direct conflict during adaptation to a visuomotor rotation.

Adaptation to visuomotor rotation is a form of implicit motor learning: it proceeds incrementally (Krakauer et al., 2000), shows limited generalization (Krakauer et al., 2000), and produces large and prolonged aftereffects when subjects are returned to baseline (Caithness et al., 2004). Nevertheless, the presence of a cognitive strategy has been invoked to explain unexpected results in rotation adaptation experiments, such as generalization (Imamizu et al., 1995; Baraduc and Wolpert, 2002) and the absence of significant aftereffects (Buch et al., 2003). The underlying assumption, which to our knowledge has not been directly examined, is that explicit strategies can substitute for implicit rotation learning. Here, we tested subjects in a visuomotor rotation task where we informed them of the imposed rotation and told them how to "cheat" by aiming to a neighboring target whose direction would exactly cancel the rotation. The success of the explicit strategy, and its substitution for adaptation, would be evident both by an abrupt stepwise cancellation of error rather than incremental adaptation and the absence of aftereffects. 


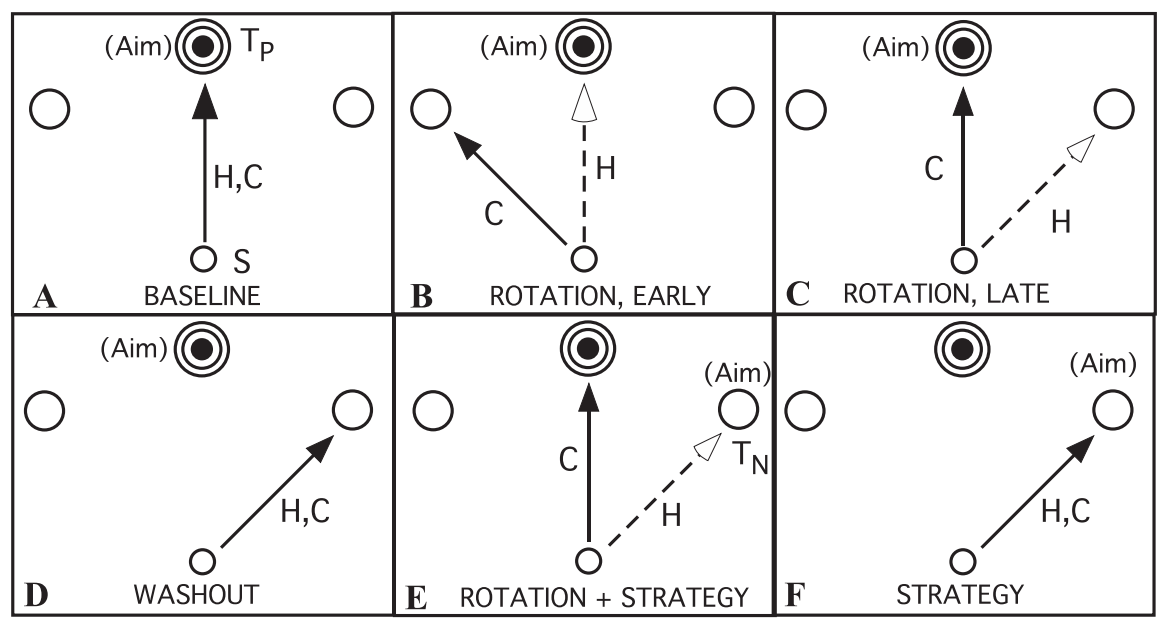

Figure 1. Task conditions. Each frame shows the start circle $(S)$ and three of the eight surrounding targets. The bull's-eye pattern indicates the target proper $\left(T_{p}\right)$, and the two open circles are the neighboring targets, $45^{\circ}$ away. The arrows indicate the direction of hand and cursor movements ( $\mathrm{H}$ and $\mathrm{C}$, respectively). $\boldsymbol{A}$, Baseline. $\boldsymbol{B}$, Early rotation $\left(45^{\circ} \mathrm{CCW}\right)$. C, Late rotation. $\boldsymbol{D}$, Washout. $\boldsymbol{E}$, Rotation plus strategy. $\boldsymbol{F}$, Strategy only. $\ln \boldsymbol{A}-\boldsymbol{D}$, subjects aim for $T_{\mathrm{p}}$. $\ln \boldsymbol{E}$ and $\boldsymbol{F}$, subjects aim for $\mathrm{T}_{\mathrm{N}}$.

\section{Materials and Methods}

Subjects. Eighteen right-handed subjects (nine men and nine women; $18-55$ years of age) participated in the study. All of the subjects were naive to the purpose of the experiments, signed an institutionally approved consent form, and were paid to participate. Subjects were randomly assigned to one of two groups.

General experimental procedure. Subjects sat facing a computer monitor with their right forearm splinted on a tripod and controlled a screen cursor by rotating their hand (held closed into a fist with surgical adhesive tape) up and down, side to side, and diagonally around the wrist. A Qualysis (Gothenburg, Sweden) Proreflex infrared camera recorded pointing direction, defined as the position of a spherical infraredreflective marker attached to a ring placed around the index finger's proximal interphalangeal joint, at a sampling rate of $100 \mathrm{~Hz}$. The target set consisted of eight radially arrayed circles, separated in direction by $45^{\circ}$, placed on a circle of radius $10 \mathrm{~cm}$ on the computer screen, which required a $2.2 \mathrm{~cm}$ movement of the infrared marker. In other words, the knuckle of the index finger had to translate $2.2 \mathrm{~cm}$ to move the cursor from the center of the circle to one of the targets. The targets were always visible. At the start of each trial, the target to be aimed for turned into a bull's-eye in synchrony with a tone. The target order was random, and there was no restriction on reaction time. Subjects were instructed to make straight out-and-back movements, to reverse direction sharply in the target, and to not make any trajectory corrections. For each movement, the cursor indicating hand position was frozen $100 \mathrm{~ms}$ after movement onset, and a white square appeared at the movement reversal point. Subjects were instructed to place both the cursor and the white square in the target, which clamped movement speed and minimized feedback corrections.

Conditions. There were four conditions. In baseline, wrist movements were mapped normally to the motion of the screen cursor (right-left and up-down were the same for the wrist and screen cursor). In rotation, the screen cursor was rotated $45^{\circ}$ counterclockwise (CCW) around the center of the start location. In rotation plus strategy, the same $45^{\circ}$ rotation was imposed but subjects were told about the rotation and instructed to counter it by aiming at the clockwise (CW) neighboring target $\left(\mathrm{T}_{\mathrm{N}}\right)$ rather than the proper target $\left(\mathrm{T}_{\mathrm{P}}\right)$. In strategy-only, subjects aimed at $\mathrm{T}_{\mathrm{N}}$ rather than $T_{P}$. As there was no rotation imposed, the cursor entered $T_{N}$. Figure 1 shows the anticipated hand and cursor trajectories for baseline, early rotation, late rotation, rotation plus strategy, and strategy-only.

Data analysis. We used the directional error at the reversal point as the measure of rotation adaptation. This was calculated as the difference between the direction of the target from the initial hand position and the direction of the hand at the movement reversal point. Wilcoxon rank sum tests were used throughout, with a significance threshold at $p<0.05$.

\section{Results}

Subjects failed to counter the rotation adaptation with an explicit strategy

Group 1 attempted to learn the rotation using a strategy and performed the sequence of conditions in Figure 2A. After a block of baseline movements (phase I), they made two movements of rotation (phase II), which resulted in the expected errors $\sim 45^{\circ}$ (Fig. $2 A$ ). Then, subjects were told the following: "You just made two large errors because we imposed a rotation that pushes you $45^{\circ}$ counterclockwise. You can counter the error by aiming for the neighboring clockwise target, which is also at $45^{\circ}$." They were thus asked to "cheat" the imposed rotation by adopting an explicit cognitive strategy (rotation plus strategy). Rotation plus strategy was initially effective as it cancelled the visuomotor rotation and subjects' errors immediately returned to near zero (Fig. $2 \mathrm{~A}$, early in phase III). However, as subjects continued to make movements in rotation plus strategy, they unexpectedly made increasingly large directional errors, leading the cursor away from $\mathrm{T}_{\mathrm{P}}$ and toward $\mathrm{T}_{\mathrm{N}}$ (Fig. $2 \mathrm{~A}$, remainder of phase III). This suggested that subjects were progressively learning the rotation around $\mathrm{T}_{\mathrm{N}}$ at the cost of not fulfilling the task requirement to be accurate to $T_{\mathrm{P}}$.

To demonstrate that subjects were indeed learning the rotation implicitly around $\mathrm{T}_{\mathrm{N}}$, they were instructed to stop using a strategy and aim at $\mathrm{T}_{\mathrm{P}}$ (i.e., switch to rotation) (Fig. $2 \mathrm{~A}$, phase IV). There was a CCW directional error, but it was significantly smaller than the expected $45^{\circ}$ (mean $\pm \mathrm{SE}, 19 \pm 3.8^{\circ} ; p<$ 0.0001 ), which indicated that the rotation had been partially learned. Finally, subjects were informed that the rotation had been switched off and instructed to aim for $T_{P}$ (i.e., washout). Substantial and long-lasting aftereffects were apparent, additional proof that implicit learning of the rotation had occurred (Fig. 2A, phase V). Thus, group 1 failed to sustain a strategy to counter the rotation at $T_{P}$ but instead learned it implicitly at $T_{N}$.

\section{The rate of rotation adaptation was not reduced by an opposing explicit strategy}

To test whether implicit learning of the rotation to $T_{N}$ engaged the same system used when subjects learn implicitly to $T_{P}$, group 2 performed the rotation condition (Fig. $2 \mathrm{~B}$ ). Critically, the mean value of the directional error over the first 24 movements (of phase III for group 1 and phase II for group 2) was not significantly different for the two groups (30.4 vs 34.9\%; $p=0.223$ ). Thus, remarkably, the initial time course of rotation learning was independent of whether subjects were concomitantly engaged in an opposing explicit strategy. It should nevertheless be noted that the amount of rotation learning was significantly greater for rotation (group 2) (Fig. $2 \mathrm{~B}, \Delta \mathrm{R}$ ) than for rotation plus strategy (group 1) [Fig. $2 A, \Delta(\mathrm{R}+\mathrm{S})$ ] (70.0 vs $42.3 \%$; $p<0.005$ ). This difference resulted because the two groups' learning curves diverged in late learning (compare Fig. $2 A$, late phase III; $B$, late phase II), when trial-to-trial variability increased significantly for rotation plus strategy, perhaps reflecting subjects' unease with their worsening accuracy to $T_{P}$ and a search for alternative strategies. In addition, the average aftereffect was not significantly different between the two groups $\left(-14.4^{\circ}\right.$ for group $1,-16.5^{\circ}$ for group $2 ; p=0.261)$. Thus, although the initial aftereffect was 

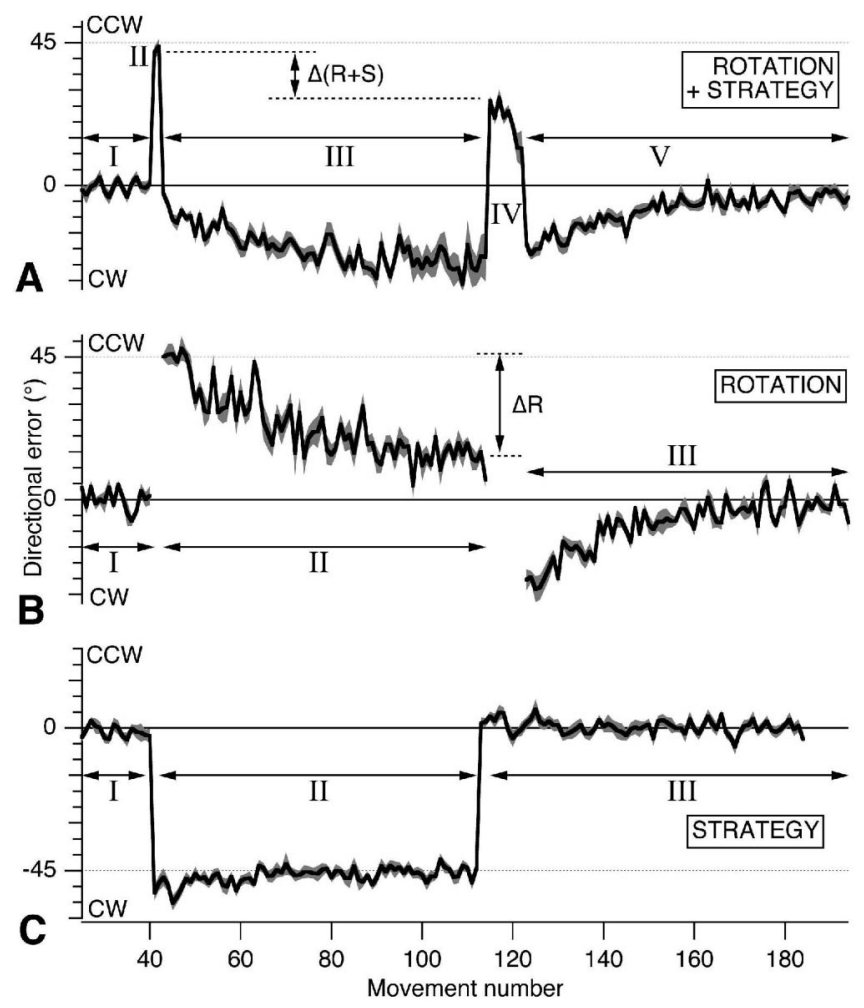

Figure 2. Time course of directional error (mean $\pm \mathrm{SE}$; in degrees) at the endpoint for each group. $\boldsymbol{A}$, Rotation plus strategy. $\boldsymbol{B}$, Rotation. $\boldsymbol{C}$, Strategy. Roman numerals indicate changes in experimental conditions (see Results for details).

smaller for rotation plus strategy compared with rotation $(-18.5$ vs $-25.4^{\circ} ; p<0.005$ ), it showed a similar time course (compare Fig. $2 A$, phase $V ; B$, phase III).

An alternative measure of the amount of adaptation that could have been considered is the difference between directional error at the beginning and end of the training period (i.e., phase III for the $\mathrm{R}+\mathrm{S}$ group and phase II for the $\mathrm{R}$ group). However, we avoided comparing these measures across the two groups, because the conditions during learning were not equivalent: during phase III, $\mathrm{R}+\mathrm{S}$ subjects were applying a cognitive strategy while experiencing a rotation, whereas during phase II, R subjects were only experiencing a rotation without using any strategy. The measure we chose, in contrast, compared subjects' errors when they were in the same behavioral state.

Subjects in group 1 were interviewed after the experiment, and they all described frustration at the fact that, despite their explicit strategy, they nevertheless got progressively worse at making the cursor hit $\mathrm{T}_{\mathrm{P}}$. They were, without exception, completely unaware of the nature of the directional errors they had been making to $\mathrm{T}_{\mathrm{N}}$.

As a final control, we examined whether aftereffects can also result from a failure to abruptly stop using an explicit strategy. Subjects in group 1 were tested on a strategy-only condition. After performing a block of baseline movements, they were instructed to aim their movements toward $\mathrm{T}_{\mathrm{N}}$ although the bull'seye was in $T_{P}$. As no rotation was imposed, this resulted in the cursor hitting $\mathrm{T}_{\mathrm{N}}$. Subjects had no difficulty immediately switching to this strategy (Fig. $2 C$, phase II), and there was no aftereffect when they switched back to baseline (Fig. 2C, phase III). In other words, when subjects used a purely cognitive strategy in the absence of the rotation, they were able to switch back to baseline within one movement. This confirmed that explicit strategies themselves do not lead to aftereffects, reinforcing the notion that aftereffects imply that implicit learning has occurred.

\section{Discussion}

The experiments presented here sought to investigate the impact of a cognitive strategy on implicit motor adaptation. The experimental paradigm was to place explicit and implicit processes in conflict during adaptation to a visuomotor rotation. Subjects were made aware of the rotation and instructed to cancel it by aiming at a neighboring target, $\mathrm{T}_{\mathrm{N}}$, to hit the proper target, $\mathrm{T}_{\mathrm{P}}$. Although this initially allowed subjects to successfully counter the rotation without the need for incremental adaptation, they were unable to sustain this situation. Instead, they adapted to the rotation around $\mathrm{T}_{\mathrm{N}}$, at the expense of increasing errors around $\mathrm{T}_{\mathrm{P}}$. The initial rate of rotation adaptation around $\mathrm{T}_{\mathrm{N}}$ in the presence of a strategy directed at $\mathrm{T}_{\mathrm{P}}$ was not different from a control group of subjects who adapted around $\mathrm{T}_{\mathrm{P}}$ in the absence of a strategy. Thus, motor learning proceeded around $\mathrm{T}_{\mathrm{N}}$, although errors were not attended to, and this led to increasing errors around $\mathrm{T}_{\mathrm{P}}$, the target on which attention was focused during implementation of the cognitive strategy.

The first finding in this study was that the cognitive strategy was initially effective, but then subjects started to make increasingly large directional errors to the desired target. On questioning after the experiment, subjects were unable to characterize the nature of their errors beyond an awareness that they made progressively larger errors to the desired target. Subjects were unaware that they became increasingly accurate to $\mathrm{T}_{\mathrm{N}}$. Thus, by verbal report, subjects were attending to the directional error around $\mathrm{T}_{\mathrm{P}}$ and not around $\mathrm{T}_{\mathrm{N}}$. This suggests that they failed to explicitly counter the effect of the rotation on performance to $T_{P}$ and simultaneously succeeded to implicitly adapt to the rotation around $\mathrm{T}_{\mathrm{N}}$. However, there have been criticisms of the use of verbal reports of awareness to prove that a process is unconscious (St. John and Shanks, 1997). These include the concern that subjects might be aware of features that are not addressed by the question (i.e., the question might not be as sensitive as the explicit awareness system itself). The novelty of our task design makes it resistant to this criticism. If subjects were indeed explicitly reducing their errors around $\mathrm{T}_{\mathrm{N}}$, then they should not have been surprised by their errors around $\mathrm{T}_{\mathrm{P}}$, but they all were.

The second finding in this study was that the initial rate of rotation learning around $\mathrm{T}_{\mathrm{N}}$ in the presence of a competing explicit strategy around $\mathrm{T}_{\mathrm{P}}$ (group 1) was not significantly different from rotation learning around $\mathrm{T}_{\mathrm{P}}$ in the absence of a concurrent explicit strategy (group 2). In other words, the cognitive strategy to $\mathrm{T}_{\mathrm{P}}$ did not interfere with adaptation around $\mathrm{T}_{\mathrm{N}}$. This result is congruent with definitions of implicit learning based on nonintention/automaticity rather than on unconsciousness/unawareness (Frensch, 1998). As outlined in Introduction, implicit learning is inferred when it is not interfered with by another task in a dual-task design. Our result can be interpreted in the same way, although, strictly speaking, we did not impose a dual-task structure, because we only asked subjects to do one thing: aim for the neighboring target to get the cursor into the proper target. Thus, we show evidence for implicit learning of the rotation both in terms of unawareness and in terms of resistance to interference by the explicit strategy. All told, these results suggest that rotations are learned purely implicitly, a conclusion reinforced by the fact that the rate of reduction of the aftereffect was not different in the two groups. These results also suggest that, unlike in the serial reaction time (SRT) task, where explicit awareness can enhance implicit learning, such synergy is not possible for rotation learn- 
ing. Functional imaging may provide a clue as to the neural substrates underlying this difference. In a recent study, similar areas, mainly in the left hemisphere, were activated during implicit and explicit learning of the SRT task (Willingham et al., 2002). In addition, activation in left prefrontal cortex was associated with explicit awareness. In contrast, we, and others, have shown that implicit rotation learning is associated with activations in the right hemisphere (Inoue et al., 1997; Ghilardi et al., 2000; Krakauer et al., 2004). Thus, for rotation learning, implicit and explicit processes may compete, because they are mediated by separate circuits in the right and left hemispheres, respectively.

Our results have two important implications for theories of motor planning and motor learning. First, they suggest that the motor system cannot tolerate a situation in which planned and executed trajectories in visual space are different, even if this is consciously chosen as the task goal. This can be explained using the framework of computational motor control (Kawato, 1999). In this framework, an inverse model computes the motor commands necessary to produce a desired trajectory, as planned in visual coordinates, whereas a forward model predicts the trajectory that will result from these motor commands. In the context of our study, the explicit goal of the task is to move the cursor into the target. When given explicit instructions on how to counteract a $45^{\circ}$ rotation, we believe the following sequence of events takes place. The motor system plans a trajectory in visual space to the neighboring target. This desired trajectory is fed to the inverse model to generate motor commands that will result in this visual trajectory. The forward model then predicts that the resulting cursor's trajectory will go to the neighboring target. Instead, the cursor goes to the true target, $45^{\circ}$ away. Thus, there is a conflict between the trajectory predicted by the forward model and the actual trajectory observed. We propose that it is this conflict that is intolerable to the motor system and that drives implicit adaptation, in opposition to the explicit strategy.

Second, the results show that the motor system can reduce errors around a target even when accuracy to that target is not the goal of the task, the errors are not explicitly detected, and attention is focused on errors to another target. This is distinct from adaptation to incremental rotations (Kagerer et al., 1997; Klassen et al., 2005), where subjects, although not aware of the systematic nature of their small errors, explicitly try to be accurate to the target they are aiming for and do not focus attention elsewhere.

We conclude that adaptation to visuomotor rotation is independent of explicit strategies, and that the motor system overrides an explicit strategy because of the need to resolve any conflict between the predictions of a forward model and visual feedback. Sports coaches should take note that, when it comes to motor learning, the brain has a mind of its own.

\section{References}

Baraduc P, Wolpert DM (2002) Adaptation to a visuomotor shift depends on the starting posture. J Neurophysiol 88:973-981.
Buch ER, Young S, Contreras-Vidal JL (2003) Visuomotor adaptation in normal aging. Learn Mem 10:55-63.

Caithness G, Osu R, Bays P, Chase H, Klassen J, Kawato M, Wolpert DM, Flanagan JR (2004) Failure to consolidate the consolidation theory of learning for sensorimotor adaptation tasks. J Neurosci 24:8662-8671.

Frensch PA (1998) One concept, multiple meanings: on how to define the concept of implicit learning. In: Handbook of implicit learning (Stadler M, Frensch PA, eds), pp 47-104. Thousand Oaks, CA: Sage.

Gabrieli JD, Corkin S, Mickel SF, Growdon JH (1993) Intact acquisition and long-term retention of mirror-tracing skill in Alzheimer's disease and in global amnesia. Behav Neurosci 107:899-910.

Ghilardi M, Ghez C, Dhawan V, Moeller J, Mentis M, Nakamura T, Antonini A, Eidelberg D (2000) Patterns of regional brain activation associated with different forms of motor learning. Brain Res 871:127-145.

Hatada Y, Miall RC, Rossetti Y (2005) Two waves of a long-lasting aftereffect of prism adaptation measured over 7 days. Exp Brain Res 169:417-426.

Imamizu H, Uno Y, Kawato M (1995) Internal representations of the motor apparatus: implications from generalization in visuomotor learning. J Exp Psychol Hum Percept Perform 21:1174-1198.

Inoue K, Kawashima R, Satoh K, Kinomura S, Goto R, Sugiura M, Ito M, Fukuda H (1997) Activity in the parietal area during visuomotor learning with optical rotation. NeuroReport 8:3979-3983.

Kagerer FA, Contreras-Vidal JL, Stelmach GE (1997) Adaptation to gradual as compared with sudden visuo-motor distortions. Exp Brain Res 115:557-561.

Kawato M (1999) Internal models for motor control and trajectory planning. Curr Opin Neurobiol 9:718-727.

Klassen J, Tong C, Flanagan JR (2005) Learning and recall of incremental kinematic and dynamic sensorimotor transformations. Exp Brain Res 164:250-259.

Krakauer JW, Pine ZM, Ghilardi MF, Ghez C (2000) Learning of visuomotor transformations for vectorial planning of reaching trajectories. J Neurosci 20:8916-8924.

Krakauer JW, Ghilardi MF, Mentis M, Barnes A, Veytsman M, Eidelberg D, Ghez C (2004) Differential cortical and subcortical activations in learning rotations and gains for reaching: a PET study. J Neurophysiol 91:924-933.

Nissen M, Bullemer P (1987) Attentional requirements of learning: evidence from performance measures. Cognit Psychol 19:1-32.

Reber PJ, Squire LR (1998) Encapsulation of implicit and explicit memory in sequence learning. J Cogn Neurosci 10:248-263.

Redding GM, Rader SD, Lucas DR (1992) Cognitive load and prism adaptation. J Mot Behav 24:238-246.

Sakai K, Kitaguchi K, Hikosaka O (2003) Chunking during human visuomotor sequence learning. Exp Brain Res 152:229-242.

Shadmehr R, Brandt J, Corkin S (1998) Time-dependent motor memory processes in amnesic subjects. J Neurophysiol 80:1590-1597.

St. John M, Shanks D (1997) Implicit learning from an information processing standpoint. In: How implicit is implicit learning? (Berry D, ed), pp 162-194. Oxford: Oxford UP.

Tranel D, Damasio AR, Damasio H, Brandt JP (1994) Sensorimotor skill learning in amnesia: additional evidence for the neural basis of nondeclarative memory. Learn Mem 1:165-179.

Willingham DB (2001) Becoming aware of motor skill. Trends Cogn Sci 5:181-182.

Willingham DB, Nissen MJ, Bullemer P (1989) On the development of procedural knowledge. J Exp Psychol Learn Mem Cogn 15:1047-1060.

Willingham DB, Salidis J, Gabrieli JD (2002) Direct comparison of neural systems mediating conscious and unconscious skill learning. J Neurophysiol 88:1451-1460. 